\title{
Physics teachers' framings of the relationship between equity and antiracism
}

\author{
Tra Huynh \\ Physical Science Division, School of STEM, University of Washington Bothell, 18115 Campus Way NE, Bothell, WA $98011-8246$ \\ Lauren C. Bauman \\ Department of Physics, University of Washington, Box 351560, Seattle, WA 98195-1560 \\ Amy D. Robertson \\ Department of Physics, Seattle Pacific University, 3307 Third Ave W, Seattle, WA, USA, 98119-1997 \\ Rachel E. Scherr \\ Physical Science Division, School of STEM, University of Washington Bothell, 18115 Campus Way NE, Bothell, WA $98011-8246$
}

With the ongoing antiracism movement in the United States, there is a call for physics teachers to incorporate equity-based and antiracist activities and curricula into their classrooms. In an online summer professional development course for high school physics teachers, we listened to participants define and compare antiracism and equity. We identified three framings (dual, part-whole, and developmental) that characterize these high school physics teachers' conceptions of the relationship between equity and antiracism. The framings offer insights into physics teachers' notions of anti-racist practice in relation to equity and their concerns regarding enacting equity and antiracism in teaching practice.

\footnotetext{
2021 PERC Proceedings edited by Bennett, Frank, and Vieyra; Peer-reviewed, doi.org/10.1119/perc.2021.pr.Huynh

Published by the American Association of Physics Teachers under a Creative Commons Attribution 4.0 license.

Further distribution must maintain the cover page and attribution to the article's authors.
} 


\section{BACKGROUND}

The practice of addressing social justice in the classroom is increasingly gaining momentum within K-12 and higher education, including in physics $[1,2]$. Two of the common approaches that we discuss in this paper are an equity lens and an antiracist lens. We use the word "lens" to indicate that equity and antiracism are frameworks through which teachers perceive and interpret their work. One explanation of equity that has been marshaled extensively within education is that equity has been achieved when everyone has fair opportunities to learn and succeed [3]. Being antiracist is, more specifically, battling racism - a system of policies and ideas that produce and normalize racial inequity [4]. Although both the equity lens and the antiracist lens work toward justice for people of all races, equity and antiracism, by these definitions, require different kinds of action and conversation; they inspire and motivate different responses. An equity lens advocates for enacting policies and practices that redistribute resources so that everyone has access to the same opportunities. Meanwhile, an antiracist lens advocates for dismantling white supremacist systems and sharing power so that people of all races can live a full and meaningful life.

Across education levels, resources for equity range from strategies for inclusion, embracing student identity, improving epistemic beliefs, to creating active-learning environments where teachers yield more control and agency to students for their own learning, etc. [5]. Resources for antiracism in physics include, but are not limited to, discussions about race and racial identity development, whiteness and privilege, colonialism, implicit bias, and stereotype threat, etc. $[6,7]$. We recognize that there is not always a clear distinction between the implementation of equity and antiracism in teaching. However, in physics, while equity has been explicitly studied over the past decades, addressing racial justice is still a rare practice [2].

Emerging from legal scholarship, Critical Race Theory (CRT) helps us understand the role of racism in educational inequity. CRT asserts that race is a social construct and racism is ingrained in all of U.S. society, from the law to education to medicine [8]. Critical race theorists and educators have pointed out multiple ways in which race, in intersection with other identities, continues to account for inequity in education [9-13].

In physics, racism shows up in outcomes - e.g., in the underrepresentation of Black, Indigenous, and Latinx students [14] - and in the experiences reported by Students and Scientists of Color, who are continually harmed by a neutral and objective view of science [15]; conventional images of who does physics, which can amplify stereotype threat and implicit bias [16, 17]; and a culture of exclusion that marginalizes underrepresented students [13, 18-20]. This calls for attention to the ways in which white supremacy is operating in physics teaching and learning contexts, and thus for teacher preparation around equity and antiracism.

Teachers in different teaching contexts may vary in their interests and actions toward challenging the status quo and inequity in education. We align with Ladson-Billings and Tate [9], who state that teaching through the lens of antiracism does not mean that other identity dimensions such as class, gender, sexuality, ability, etc. are insignificant, but rather, that race matters in education.

Although some educators view equity and antiracism as inseparable [21], research has shown that there is more resistance to antiracist education and discussions about race than about equity [22], and there are equity agendas that overlook the privilege and power that whiteness affords [23]. Our study offers an explicit and focused discussion on physics teachers' ideas about the relationship between equity and antiracism in their teaching practice. The findings provide insights into physics teachers' decisions to incorporate equity and/or antiracism, as well as the challenges and barriers that physics teachers encounter when taking up the antiracist lens versus the equity lens. We come to this paper from our recognition that broaching conversations about race and equity in a classroom can be daunting - perhaps especially in a physics classroom, which can seem superficially disconnected from questions of racial justice. Even well-intentioned, fairness-committed educators can practice racism without being aware of it [24]. Therefore, our goal in this work is to contribute to the current efforts of antiracist educators and researchers to promote critical reflection and raise racial consciousness in teaching practice in order to advance social justice.

\section{METHODOLOGY}

\section{A. Context}

The data comes from a teacher professional development (PD) workshop that took place in August 2020. The workshop was a virtual one-week PD that enrolled 22 high school physics teachers (21 from the U.S., 1 from Canada). The goal of the ongoing, grant-funded PD was to support physics teachers in understanding the sociopolitical nature of the energy concept. Each day of the PD featured a different guest facilitator, two regular facilitators, and one "master teacher" with substantial experience integrating equity into physics teaching. Teachers self-reported demographic information; there were 14 females, 6 males, and 2 nonbinary people. 17 teachers identified as White/Caucasian, 2 as Black/African American, 1 as Asian, 1 as Latinx/Hispanic, and 1 as Multiracial.

The conversations we quote in this paper came from the first day of the workshop, after the group of teachers had established group norms, introduced themselves, and shared their thoughts on the question, "what is equity to you?" Towards the end of the day, one of the facilitators asked the 
teachers to respond to three prompts in breakout rooms: (i) contrast equity and antiracism, (ii) explore new visions of instruction that connect equity with physics, and (iii) make a list of things you need or want to learn more about. The participants were randomly placed into 5 breakout rooms.

This analysis foregrounds conversations from two groups, A and B. Group A consists of Leslie, Lori, Maggie, and Megan (all white females). Group B consist of Rebecca, Kelsey (both white females), Lisa (white, Hispanic female), and Tim (white male). All names are pseudonyms. We chose these two groups because the teachers in these groups were engaging in explicit and direct conversation that contrasted equity and antiracism. The data was studied by a group of authors with positionalities as follows: the first author identifies as an Asian woman who was a first-generation college student; the second author as a white woman; the third author as a disabled white woman; and the fourth author as a white-passing Jewish woman. We have each had lived experiences of spaces that are not equitable for us, in that we are not fully included in them, even though those spaces may be called equitable by others. These experiences help us understand the contrast between equity and antiracism or justice, as well as motivation to voice the importance of this kind of work in education research.

We acknowledge that our findings are limited to white teachers' perspectives; (the majority of teacher participants we analyzed here are white). At the same time, the majority of high school physics teachers are white: statistics by AIP show that white teachers made up $92 \%$ of all physics teachers in U.S. high schools in 2008 - 2009 [25], which may make it even more significant to engage in the conversation with teachers and within our educator community around physics teachers' practice of equity and antiracism. We neither claim that these findings are generalizable, nor argue that they fit for all white teachers and teachers of all races. Instead, we want to contribute this work as a basis for further explicit, principled, and direct conversations with our community around equity and social justice.

\section{B. Thematic analysis}

We conducted a thematic analysis with an inductive approach [26]. Thematic analysis offers a method for analyzing, identifying, and interpreting emergent meaning from qualitative data, which is an appropriate tool for our exploratory qualitative research. We followed a 6-step iterative process to conduct our thematic analysis [26, 27]. We familiarized ourselves with the data while transcribing the videos, reading field notes, and debriefing with other researchers. This was when we noticed the episodes featuring discussion of equity and antiracism and decided to focus on two groups of teachers where the conversations were rich and explicit. Then, the first author generated initial codes identifying the relationship between equity and antiracism, applied inductive coding to the transcripts lineby-line, and searched for emergent themes. The initial emergent themes were reviewed among the authors and were shared with one facilitator of the summer workshop. The authors proceeded through multiple rounds of revising the coding schemes and the themes. This iterative process resulted in a final set of themes that were distinctive and collectively covered the whole data set.

\section{RESULTS}

We found three emergent themes in the teachers' conversations comparing the equity and antiracist lenses: dual framing, part-whole framing, and developmental framing. Dual framing contrasts equity and antiracism, whereas part-whole framing and developmental framing treats equity and antiracism as subcategories and on a spectrum, respectively, in teaching practice.

\section{A. Dual framing}

Dual framing treats equity and antiracism as two distinct approaches with their own sets of characteristics, perceptions, implications, and goals. Our goal in naming dual framing is to orient the audience to some of the axes on which the teachers locate the tensions between antiracism and equity, including the advantages and disadvantages they perceive in each.

For example, in group B, Tim put equity and antiracism lenses on the personal to structural axis, comparing where equity and antiracism fall, in structural versus individual spheres.

Tim: In my head, equity feels personal. It is how am I helping a person or a group of people, uh, in this situation that I can control, which is my classroom. Whereas antiracism work feels structural. You are attacking a system that has--, that is so complex that we need to begin dismantling it bit by bit, typically that is done in service of a person or a group of people.

Specifically, for Tim, antiracist work is structural, as antiracism is battling systemic racism. In contrast, he can enact equity in his personal relationships, for example with his students, in which he feels more agency. The axis that received the most consensus among the teachers in group $\mathrm{A}$ is that equity is associated with palatability, looking towards the future with optimism, while antiracism has a connotation of confrontation, and is focused on correcting the past. One teacher in group A, Maggie, stated:

Maggie: I feel like equity is the positive thing that we're striving for, but I also feel like there's so many different ways to look at it, that antiracism makes it a little bit more explicit. That there's things that--, there's history, there's context, there's things that need to be undone. It's not just the ideal of what we're trying to achieve. It's--, there's things that we're fighting against. That's not--, it's not a blank slate that we're starting from.

Here, Maggie emphasizes that taking up the antiracist lens is acknowledging the reality of racism and battling it, where equity is "the positive thing." Additionally, the 
teachers agreed that the lens of equity is general, whereas the lens of antiracism is specific. In group B, Kelsey emphasized antiracism as a SMART goal (Specific, Measurable, Attainable, Realistic, and Time-bound) [28] whereas equity "feels bigger" and "more overwhelming to tackle as a whole." Group A suggested that the vastness and vagueness of the equity lens makes incorporating equity into teaching safer or more palatable. However, for some teachers such as Maggie and Leslie, it means that the equity lens has significant drawbacks in practice. Following Maggie's (underlined) critique above, Leslie said:

Leslie: Maggie, I agree with you. I think like equity is one of those statements that you would see in like a school, like their mission statement: 'We aim to have an equitable learning environment for all of our students,' and then it gives you no idea of how to get there. [Long pause.] Although I can imagine that my very, very white school, if we said, 'we're going to have an anti-racist environment' would have tuition just [gesture indicating a drop].

In this statement, Leslie suggests that the equity lens is so vague that schools can commit to equity yet offer little actionable direction to achieve it. Meanwhile, the antiracism lens offers a clear vision of the basis on which one can make explicit judgement and criticism of whether antiracist work is being enacted, i.e., prohibitively high tuition. Then, Maggie added:

Maggie: It's like equity is--, uh, it feels like a safer place to start, but I don't know if that's a good thing or not. I don't know if safety should be the priority. [...] I teach in a school where, when, if I--, when I raise these issues, there is pushback from a significant percentage of people, students, families. And so my inclination is, 'Oh yeah, I should say this in a safe, friendly way', but then there's a lot more room for not being--, like using the same word, but not talking about the same thing. Because everybody's got their own image of what equity is. And it's kind of this feel-good phrase, but I don't know.

Comparing across the two axes of palatability and generality, Maggie asserts that the drawbacks of equity lens outweigh the comfort that it offers in practice. The dual framing primes the teachers to view the equity approach and the antiracism approach as separate and in tension with each other. Although equity, with its general characteristics, seems easier in practice than antiracist work, the equity lens may also lack direction or accountability.

\section{B. Part-whole framing}

Part-whole framing emerges when the teachers treat equity as inclusive of antiracism, or antiracist work as part of an equity approach. Kelsey [group B] used an analogy in which equity was a rectangle and antiracism was a square (which is a specific type of rectangle). Tim further explained:

Tim: For me, equity encompasses quite a bit more than anti-racist work. Um, mostly because anti-racist work by its definition is focused solely on race. Whereas equity work can certainly be race-focused, but it can also be sex-focused, gender-focused, age-focused, abilityfocused. I mean, we can, we can look at it through a myriad of lenses. And, and so, uh, I think that anti-racist work is inherently equity work. I also don't think all equity work will be anti-racist.

According to Tim, equity work addresses multiple social identities, not just race, which suggests viewing antiracist work as an element under the equity umbrella. Importantly, Tim emphasizes that not all equity work is antiracist work, depending on the type of marginalization an equity approach focuses on. In group A, Lori took up the part-whole framing when she compared the order in which equity and antiracism are approached:

Lori: Um, if you put equity first, you can kind of prevent having to pull antiracism behind you, right? So I think if, if your focus could really truly be on equity, then, um, like Megan said, I, I think for a lot of people, when you say racism, people right away, put up a flag and say, 'well, that's not me. I'm not one of those, right? And so, then you have to battle that on top of the fact that you have to- and you want to-- provide resources for everyone wherever they're at. So, if you could have equity first, then you wouldn't have to address antiracism.

In this statement, Lori expresses that tackling racism first can create difficulties due to people's defensiveness about racism. Lori suggests that by focusing on creating an equityoriented classroom, antiracism will automatically be addressed as a byproduct of achieved equity. Lori's suggested practice asserts that that antiracism is inherently included in equity, which reflects part-whole framing.

Part-whole framing can be marshaled in ways that are harmful. In particular, harm can come from using this framing to suggest that in practicing equity, one does not have to be intentional about antiracism or can skip addressing racism (because, as equity is achieved, racism will be automatically extinguished). Although equity approaches work to close the achievement gap across groups of students, advancing justice requires that educators understand the impact of racism in marginalization and address injustice through the lens of race in intersection with other identities $[8,9]$.

\section{Developmental framing}

Developmental framing conceptualizes antiracist work as a significant step towards equity work, advocating for being antiracist while working toward equity. In this framing, teachers refer to antiracism as a precondition of equity. For example, Leslie stated:

Leslie: [...] I think that antiracism is like a stepping stone towards equity, because it's something, that, like, we have to undo the things that are binding us before we can be fully there and present and accessible and all of the things that we're hoping for when we get to equity. 
In this statement, Leslie expresses the critical order of addressing antiracism and equity: racism needs to be confronted in order to set up an orientation toward equity. In group B, Rebecca asserted:

Rebecca: [...] we have to remember to be anti-racist teachers in our search for equity in our classroom. And we have to do a whole lot more for equity in our classroom, but being anti-racist is a big piece of equity in our classroom.

Rebecca's statement is an example of developmental framing, in which antiracism is a fundamental component of equity approach. Expanding further to practice, Lisa (group B) expressed the importance of day-to-day antiracist actions important in leading to greater equity:

Lisa: [...] I feel like that [antiracist work] is something I have more agency with, and I feel like I can do anti-racist things each day in my classroom, in my personal life. And it's working, we're working towards equity and that seems like this utopia that we're all trying to get to.

In these quotes, both Rebecca and Lisa emphasize the broad goal of equity. However, they do not simply compare the goal of equity with that of antiracism as in dual framing where equity is broad and antiracism is narrower. Instead, they treat antiracism as a critical step toward advancing equity. Whereas the equity lens in the part-whole framing does not necessarily or explicitly rest on antiracism, the equity lens in the developmental framing takes on antiracism as a central pillar to addressing inequity. The teachers across group A and group B often bring out the developmental framing based on the limitation of the current equity lens and practice. The developmental framing is therefore fundamental for systematic actions toward equity, considering that equity approaches alone often lack directedness and direction.

\section{CONCLUSIONS}

Both equity work and antiracist work endeavor to secure equality for all races. Many schools and educators have responded to calls for equity by foregrounding "opportunities to learn." "Opportunity to learn" is conceptualized in terms of equal access and equal outcomes, which often translates to intentionality in designing accessible learning environments, learning materials, and assessments. But what this perspective of equity often misses is a systems-level view that addresses institutionalized racism. Critical race theory states that students are living in a white-supremacist society, and that racism reaches all corners of their lives. This includes physics classrooms, as evidenced by educational outcomes within physics [14] and experiences of marginalization and oppression reported by Students of Color $[15-18,20]$. It is critically important, then, for physics teachers to learn about and enact equitable and anti-racist instructional practice. One first step in this work is understanding what these concepts mean and could look like and reflecting on ways in which existing ideological orientations towards equity and antiracism are influencing pedagogical decisions.

In our study, we identified three framings that highlight various ways in which high school physics teachers conceptualize the relationship between antiracism and equity. Dual framing productively contrasts equity and antiracism by locating them on different characteristic dimensions. Dual framing represents physics teachers' call for equity frameworks that are actionable, direct, and explicit and their seek for antiracist practices that are safe to try in their physics classroom. Part-whole framing and developmental framing focus on the interplay between antiracism and equity but they posit very different roles for antiracism. While part-whole framing can incite practice that overlooks the importance of antiracist work, developmental framing emphasizes the significance of antiracism in advancing equity. Part-whole framing, which focuses only on equity, may leave racism -- the roots of racial inequity out of focus, allowing for inaction on racism and the preservation of racism and racial inequity in students' lives. Developmental framing, on the other hand, guides practitioners to intentionally attend to racism in working towards equity. Therefore, developmental framing aligns with CRT, advocating for true equity that stems from recognizing and combatting systemic racial injustices [20].

Challenges in integrating an antiracist lens come not only from the complex structure of antiracism itself, but also from potentially hostile reactions from schools, parents, and students. Teaching is no longer, and never was, a politically neutral act $[29,30]$. Antiracism and equity are both structural work that addresses policies, processes, and systems. It is important to name the issue in order to fix it. It is important for us as researchers and educators to create opportunities for conversations among teachers, policymakers, parents, administrators, and education researchers that elicit the role of teaching in advancing social justice.

\section{ACKNOWLEDGMENTS}

We are greatly indebted to the Energy and Equity Summer Workshop 2020 organizers and teacher participants. We are thankful for the support from Tali Hairston, our research team members, and our copy editor, Sarah Kremen-Hicks. This work is indebted to the intellectuals and social activists in the education community advocating for systemic changes. This material is based upon work supported by National Science Foundation Grant No. 1936601. 
[1] https://www.aapt.org/Resources/Race-and-PhysicsTeaching.cfm Retrieved 4/30/2021.

[2] White, G. (2016). Race and physics teaching, and the fair: A call to all physics educators for manuscripts on a rarely discussed topic.

[3] Lynch, S. J. (2000). Equity and science education reform. Routledge.

[4] Kendi, I. X. (2019). How to be an antiracist. One world.

[5] National Research Council. (2012). A framework for K-12 science education: Practices, crosscutting concepts, and core ideas. National Academies Press.

[6] Daane, A. R., Decker, S. R., \& Sawtelle, V. (2017). Teaching about racial equity in introductory physics courses. The Physics Teacher, 55(6), 328-333.

[7] Pollock, M. (Ed.). (2008). Everyday antiracism: Getting real about race in school. The New Press.

[8] Delgado, R., \& Stefancic, J. (2017). Critical race theory: An introduction (Vol. 20). NyU press.

[9] Ladson-Billings, G., \& Tate, W. F. (2006). Toward a critical race theory of education. Critical race theory in education: All God's children got a song, 11, 30 .

[10] Howard, T. C. (2003). Culturally relevant pedagogy: Ingredients for critical teacher reflection. Theory into practice, 42(3), 195-202.

[11] Barton, A. C., \& Yang, K. (2000). The culture of power and science education: Learning from Miguel. Journal of Research in Science Teaching: The Official Journal of the National Association for Research in Science Teaching, 37(8), 871-889.

[12] Nissen, J. M., Horses, I. H. M., \& Van Dusen, B. (2021). Investigating society's educational debts due to racism and sexism in student attitudes about physics using quantitative critical race theory. Physical Review Physics Education Research, 17(1), 010116.

[13] Rosa, K., \& Mensah, F. M. (2016). Educational pathways of Black women physicists: Stories of experiencing and overcoming obstacles in life. Physical Review Physics Education Research, 12(2), 020113.

[14]

https://www.aps.org/programs/education/statistics/degre esbyrace.cfm

[15] Gutmann, B., Ochoa-Madrid, E., \& Olmstead, A. (2020) "I'm not that important": Barriers and bolsters to student agency during conversations about the intersections of physics and ethics. Physics Education Research Proceedings 2020.

[16] Rifkin, M. (2016). Addressing underrepresentation: Physics teaching for all. The physics teacher, 54(2), 72-74.

[17] Prescod-Weinstein, C. (2020). Making Black women scientists under white empiricism: the racialization of epistemology in physics. Signs: Journal of Women in Culture and Society, 45(2), 421-447.

[18] Quichocho, X. R., Schipull, E. M., \& Close, E. W. (2020) Understanding physics identity development through the identity performances of Black, Indigenous, and women of color and LGBTQ+ women in physics. Physics Education Research Proceedings 2020.

[19] Barthelemy, R. S., McCormick, M., \& Henderson, C. (2016). Gender discrimination in physics and astronomy: Graduate student experiences of sexism and gender microaggressions. Physical Review Physics Education Research, 12(2), 020119.

[20] Dickens, D., Jones, M., \& Hall, N. (2020). Being a Token Black Female Faculty Member in Physics: Exploring Research on Gendered Racism, Identity Shifting as a Coping Strategy, and Inclusivity in Physics. The Physics Teacher, 58(5), 335337.

[21] McNair, T. B., Bensimon, E. M., \& Malcom-Piqueux, L. (2020). From equity talk to equity walk: Expanding practitioner knowledge for racial justice in higher education. John Wiley \& Sons.

[22] Picower, B. (2009). The unexamined whiteness of teaching: How white teachers maintain and enact dominant racial ideologies. Race ethnicity and education, 12(2), 197-215.

[23] Wane, N. (2003). Anti-racism in teacher education: Rethinking our practice.

[24] Kailin, J. (2002). Antiracist education: From theory to practice. Rowman \& Littlefield.

[25] https://www.aip.org/statistics/data-graphics/proportion-highschool-physics-teachers-each-racial-or-ethnic-group-2008-09 Retrieved 4/30/2021

[26] Xu, W., \& Zammit, K. (2020). Applying thematic analysis to education: A hybrid approach to interpreting data in practitioner research. International Journal of Qualitative Methods, 19, 1609406920918810.

[27] Braun, V., \& Clarke, V. (2006). Using thematic analysis in psychology. Qualitative research in psychology, 3(2), 77-101.

[28] O'Neill, J. (2000). SMART goals, SMART schools. Educational Leadership, 57(5), 46-50.

[29] Rodriguez, A. J. (2016). For whom do we do equity and social justice work? Recasting the discourse about the other to effect transformative change. Interrogating whiteness and relinquishing power: White faculty's commitment to racial consciousness in STEM classrooms, 241-252.

[30] Hooks, B. (2014). Teaching to transgress. Routledge. 